\title{
Automatic Underwater Image Enhancement using Improved Dark Channel Prior
}

\author{
MARCO BLOCK, Hochschule für Technik und Wirtschaft Dresden, Germany \\ BENJAMIN GEHMLICH, Hochschule für Technik und Wirtschaft Dresden, Germany \\ DAMIAN HETTMANCZYK, Hochschule für Technik und Wirtschaft Dresden, Germany
}

\begin{abstract}
Images taken under water are often of a monochromatic appearance, due to the physical interaction (absorption and reflection) between particles and light sources. Enhanced images with improved saturation, for which the monochromatic character has been corrected, are more suitable for generating 3D models and for identifying structures and materials by human experts. In this paper, we present an automatic method to identify the mean water color from a set of images. This mean color represents an average gray and is used to describe a new axis in CIELab color space. An extended color variance and a histogram equalization are simultaneously applied to the image. The main advantage of this method is the fully automatic enhancement process. An UUV (Unmanned Underwater Vehicle) can operate without providing a color reference scheme. The presented method was implemented in the software JEnhancer, which is freely available. JEnhancer was successfully tested in several documentation campaigns, and was integrated into the videogrammetric software pipeline Archaeo3D to produce 3D models from videos.
\end{abstract}

\section{Key words:}

Image enhancement, Automatic color transformation, Underwater Archaeology, Videogrammetry, UUV.

\section{SDH Reference:}

Marco Block, et al. 2017. Automatic Underwater Image Enhancement Using Improved Dark Channel Prior. SDH, 1, 2, 566-589.

DOI : $10.14434 /$ sdh.v1i2.23214

\section{MOTIVATION AND INTRODUCTION}

Archaeologists greatly profit from technical progress in camera technology. The photogrammetric approach, in particular to catalogue single objects [Santos and Ritz 2013] or in combination with UAVs (Unmanned Aerial Vehicles) to document complete excavation areas [Remondino 2011], is an easyto-use alternative to traditional spatial sensing methods and is currently evolving toward becoming the standard documentation method.

Underwater photogrammetry depends on clear water conditions and adequate lighting. Unfortunately, the color range decreases with increasing depth [Iqbal et al. 2007]. The longer the

Corresponding author: Prof. Dr. Marco Block-Berlitz, Hochschule für Technik und Wirtschaft Dresden, Germany; email: block@htw-dresden.de

Permission to make digital or hardcopies of part or all of this work is granted without fee according to the open access policy of SDH.

(C) 2017 SDH Open Access Journal 
frequencies, the more absorption occurs. Due to absorption, the water color often seems bluish (Fig. 1a).

The method presented here using an improved Dark Channel Prior [He et al. 2011] is implemented in the software JEnhancer. It estimates the mean water color automatically and corrects the images before the $3 \mathrm{~d}$ reconstruction process is applied (Fig. 1b). Not only absorption has an influence on visual perception, but scattering is also an important factor. Depending on particle interaction, light energy will be redirected between the objects and the observer. To compensate for this effect, our software also improves image contrast.

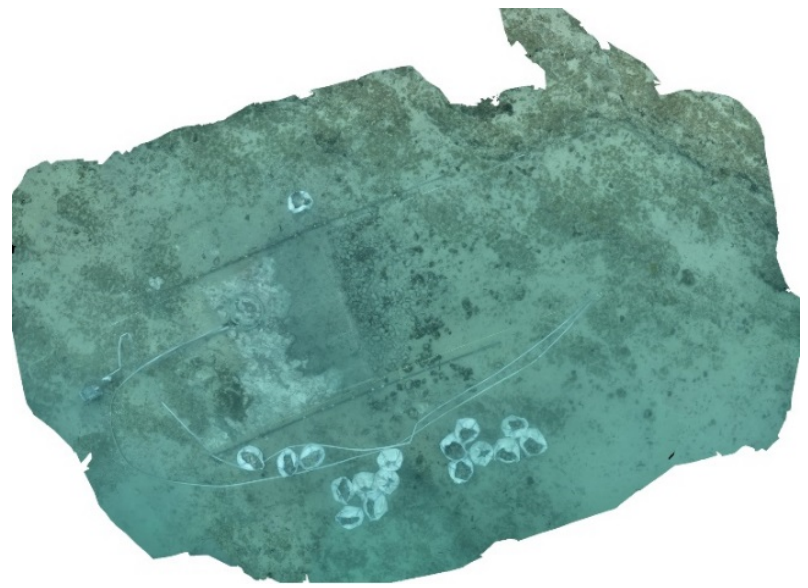

a)

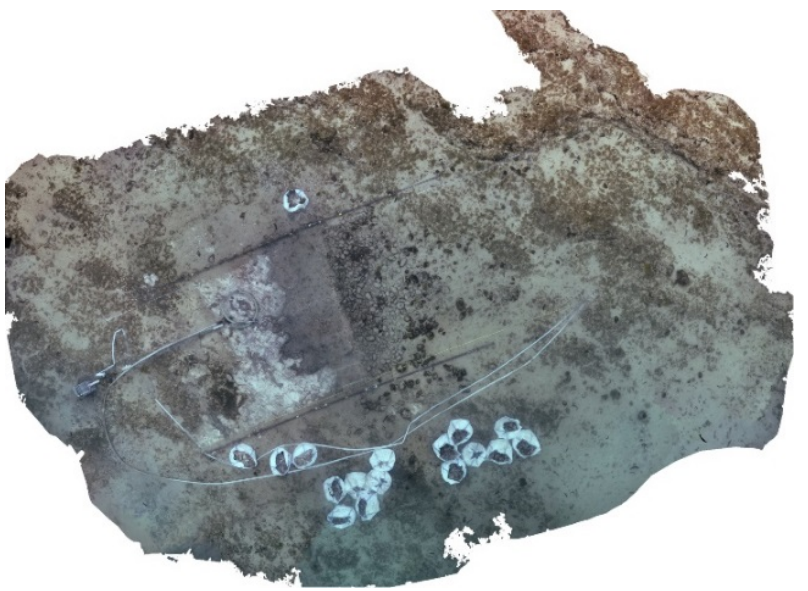

b)

Figure 1. a) The image shows an orthoimage of the campaign in Veruda/Croatia 2016 [Scholz et al. 2016], generated from the original image data taken by a GoPro Hero $4 \mathrm{BE} ; b)$ The result after, improving the input images with JEnhancer.

To improve underwater images, different restoration and image enhancement methods were developed in the past [Schettini and Corchs 2010]. This is an ongoing process, but unfortunately no free tools for practical use are currently available. This might be the reason why many archaeologists use the automatic color correction of proprietary software like Photoshop ${ }^{1}$ [Bianco et al. 2015, Bruno et al. 2015]. The tool presented here, JEnhancer, is a candidate for closing this gap.

This paper is structured as follows: first, Section 2 introduces related aspects of underwater archaeology, photo- and videogrammetry, gives a brief introduction to light properties under water and presents state-of-the-art image enhancement approaches. Next, Section 3 introduces the freely available software JEnhancer, gives some use cases and describes the internal workflow. Interim results from different campaigns and experiments are discussed in Section 4. Finally, the conclusion as well as perspectives for future work are presented in Section 5.

\footnotetext{
${ }^{1}$ http://www.adobe.com/de/products/photoshop.html
} 
1:568 M. Block et al.

\section{THEORY AND RELATED WORK}

The workflow of underwater documentation is still complex and expensive, even when laser scanning is deployed [Moisan et al. 2015]. Scientific divers need special qualifications to work under water [Papadimitriou 2015] and their diving time is limited. To reap the benefits of current technological developments in small diving robots, so-called UUVs (Unmanned Underwater Vehicles), projects like Archaeonautic ${ }^{2}$ or ArcheoROV ${ }^{3}$ were initiated. They share a focus on low-cost solutions for underwater archaeology. Beside the documentation of known archaeological sites, potential sites can be explored easier and more cost-effectively. Georeferencing under water is still a crucial challenge, because no GPS signal is directly available. To solve this, indirect solutions are often deployed [Baletti et al. 2015, Scholz et al. 2016].

\subsection{Photogrammetry and Videogrammetry in Underwater Archaeology}

The importance of 3d photogrammetry [Hartley and Zisserman 2004] in archaeology has increased [Baletti et al. 2015, Pruno et al. 2015] like few other technologies in the field in recent years. The results of terrestrial and underwater photogrammetry are quite comparable [Troisi et al. 2015]. Structure from Motion (SfM) is a popular and accurate method for deriving 3d structure from sequences of single images [Wei et al. 2013, Hartley and Zisserman 2004]. Within the SfM processing flow, feature extraction is the first step and serves to reduce all available pixels into a smaller set with relevant information. One of the most common feature detectors, used in various applications like object recognition, image retrieval and 3d reconstruction, is SIFT [Lowe 1999], published and patented by Lowe. There are different approaches to speeding up feature detection, like SiftGPU ${ }^{4}$ or related methods like SURF [Bay et al. 2008]. But in robotics, when computer vision needs to work in real-time, other solutions are more common [Miksik and Mikolajczik 2012].

As a complete $3 d$ reconstruction software, AgiSoft PhotoScan ${ }^{5}$ is widely used. Beside proprietary solutions, free software packages like VisualSFM ${ }^{6}$ and open source packages like MicMac [Deseilligny and Clery 2011], OpenMVG [Moulon et al. 2013], and Bundler [Snavely et al. 2006] are also available. In contrast to related projects, we successfully use videogrammetry [Greenwood 1999, Pappa et al. 2003, Nistèr 2004, Pollefeys et al. 2004] instead of the usual photogrammetry in aerial and underwater environments.

In the related research area of real-time robotics, an important focus is on outfitting autonomous vehicles with sensors that produce continuous 3D information about the surroundings. Laser scanning and camera-based approaches play key roles in this effort [Lime et al. 2014]. Adopting such (near) real-time approaches for videogrammetric documentation in archaeology has significant advantages, given the fact that data recording must often be completed in a small time window. Most importantly, it is possible to generate preview models and assess their quality on site. Timeconsuming processing at high detail levels can be deferred to a later time. Our experience with

\footnotetext{
${ }^{2}$ http://www.archaeonautic.de

${ }^{3}$ http://arc-team-open-research.blogspot.de/

${ }^{4}$ http://www.cs.unc.edu/ ccwu/siftgpu/

${ }^{5} \mathrm{http}: / /$ www.agisoft.ru/products/photoscan/

${ }^{6}$ http://ccwu.me/vsfm/
} 
recording data from a moving vehicle clearly shows videogrammetry to be a fault-tolerant, costeffective and easy-to-use approach [Gehmlich and Block 2015].

\subsection{The project "Archaeonautic"}

In the project Archaeocopter ${ }^{7}$, a semi-automatic software called Archaeo3D was developed to optimize and control the complete reconstruction process. The resulting $3 \mathrm{~d}$ models are strongly dependent on the quality and the correlation of the input images [Gehmlich and Block 2015]. Videos and photos are automatically imported and processed. The software is able to reorder or adjust the processing pipeline's modules and adjust the parameters, depending on the current hardware and the recording situation and complexity. VisualSFM and CMPMVS [Jancosek and Pajdla 2011] are the most important tools used by Archaeo3D.

Archaeo3D is also the key software element of the project Archaeonautic which is focused on underwater archaeology (Fig. 2).

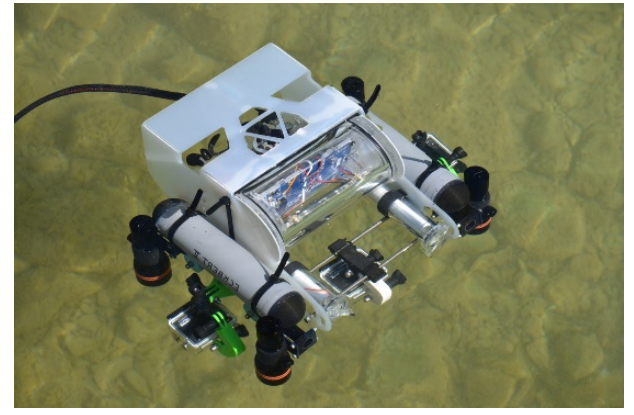

a)

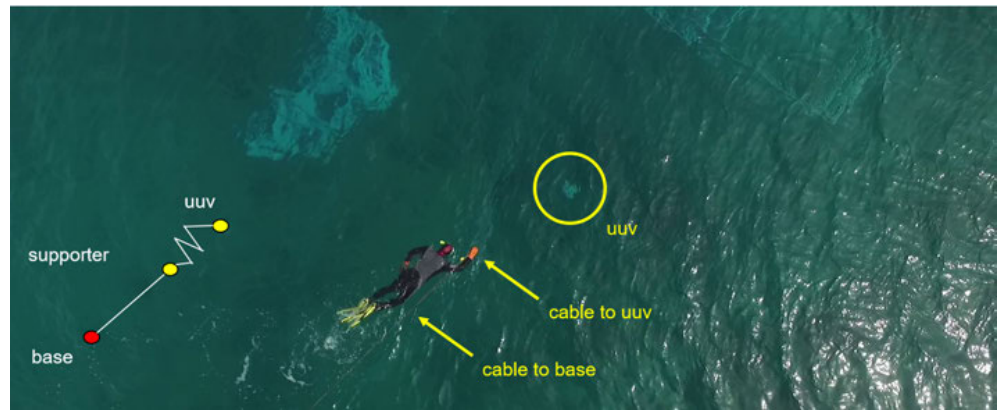

b)

Figure 2. The images show "Eckbert-II" in lake Mondsee (Austria): a) a flexible camera and lighting setup can accommodate different recording needs and environmental conditions. To document the pile dwellings in lake Mondsee, three GoPro Hero 4 BE were attached to the UUV. Subsequently, weights and buoyancy bodies were combined to balance the UUV; b) to improve the control of the UUV, a human diver supports the cable.

The project's work horse UUV, Eckbert-II, is still under development and has successfully completed campaigns in: Zwischenahner Meer/Germany (cooperation with Niedersächsisches Institut für Historische Küstenforschung), Veruda/Croatia (cooperation with DAI Frankfurt, ICUA Zadar/Croatia), and UNESCO World Heritage Site pile dwellings at lake Mondsee/Austria (cooperation with Kuratorium Pfahlbauten). The projects "Archaeocopter" and "Archaeonautic"8 (HTW Dresden and Freie Universität Berlin) were initiated in cooperation with the German Archaeological Institute (DAI) and the Archaeological Heritage Office in Saxony. The philosophy of both projects is not to maximize the level of detail achievable using current recording technology, but to focus on what is actually required for different practical purposes.

\footnotetext{
${ }^{7}$ http://www.archaeocopter.de

${ }^{8}$ www.archaeocopter.de
} 
Following this philosophy, we try to find cost-effective alternatives to established technologies in archaeology and to make them usable for everyone. The need to improve underwater images for $3 d$ reconstruction was the motivation to develop JEnhancer, which is provided free for research purposes only.

\subsection{Absorption and Scattering}

Radiative transfer theory provides the basis for understanding light propagation in water [Mobley 2001]. The quality of underwater images strongly depends on inherent optical properties [Jaffee 2001]. Temperature, salinity and the composition of water influence the attenuation of light underwater due to scattering and absorption effects throughout the light path [Berwald 1995].

Scattering changes the direction of light, depending on dissolved organic matter or small, suspended biological organisms [Stramski 2004]. It also reduces the contrast over a given distance between object and viewer. Light rays should lose their intensities punctually, but the missing part is compensated by the surrounding scattering [Cozman and Krotkov 1997]. This effect limits the range of recording distances underwater with a camera.

The absorption process reduces the light intensity between the lighting source and the photographed object and is dominated by the interaction of photons with water molecules [Schettini and Corchs 2010]. If a photon collides with a molecule, it is absorbed and converted to heat energy [Stramski 2004]. This interaction depends strongly on the light's wavelength. The longer the frequency, the stronger this attenuation. That is the reason why seawater often takes on a bluish appearance. If the concentration of organic matter and other dissolved materials is higher, the visual spectrum shifts towards green or sometimes red (Fig. 3).

Most methods focusing on underwater image enhancement deal with absorption and scattering, due to their strong impact.

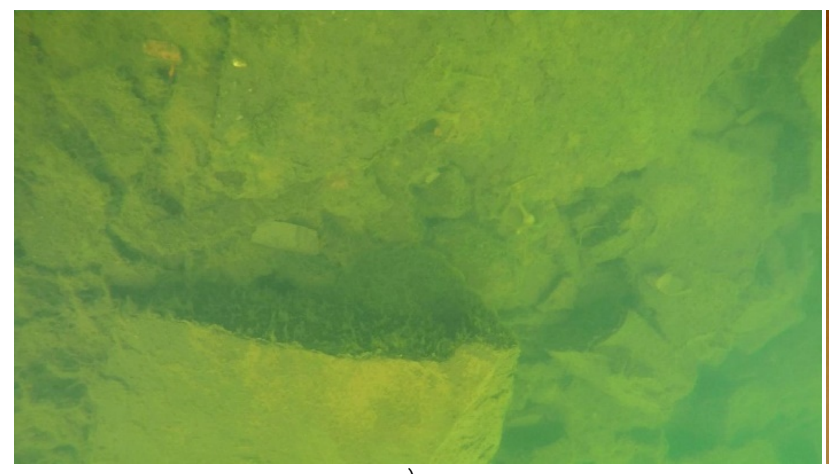

a)

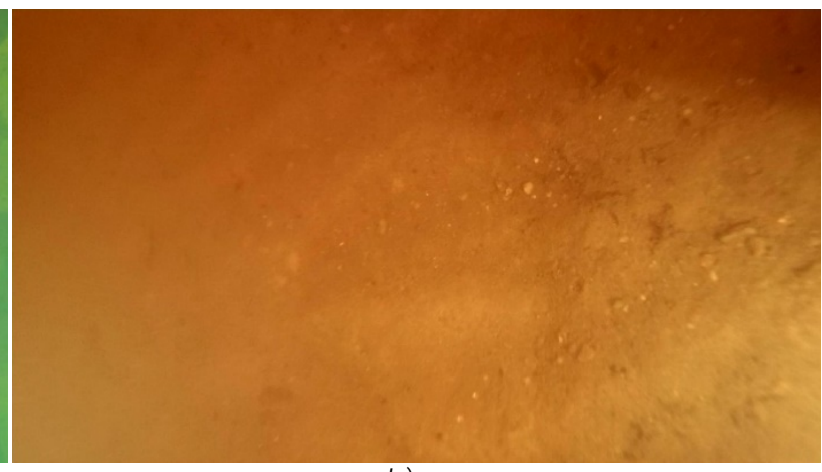

b)

Figure 3. a) this image was taken in Kamenz/Germany 2016 and looks greenish; b) in Zwischenahner Meer/Germany 2016 the specific chemical compositions delivered reddish images. 


\subsection{Automatic color correction in Photoshop}

To improve one image without having any other information, Photoshop's automatic color correction (Auto Color) can produce promising results for underwater images (Fig. 4).

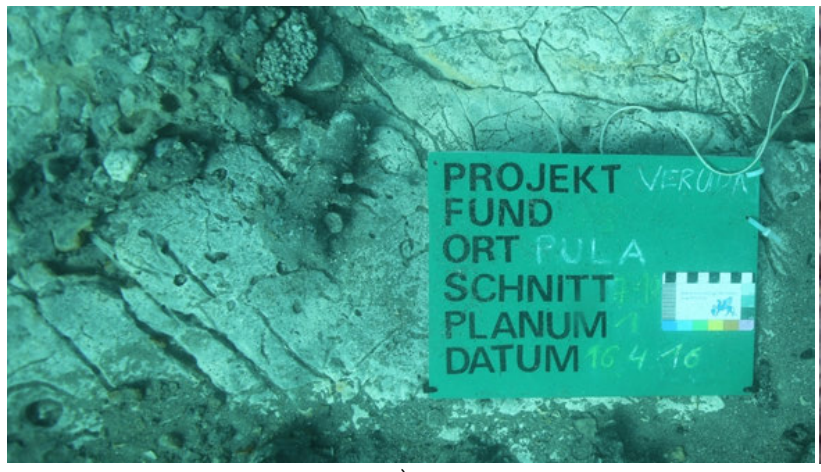

a)

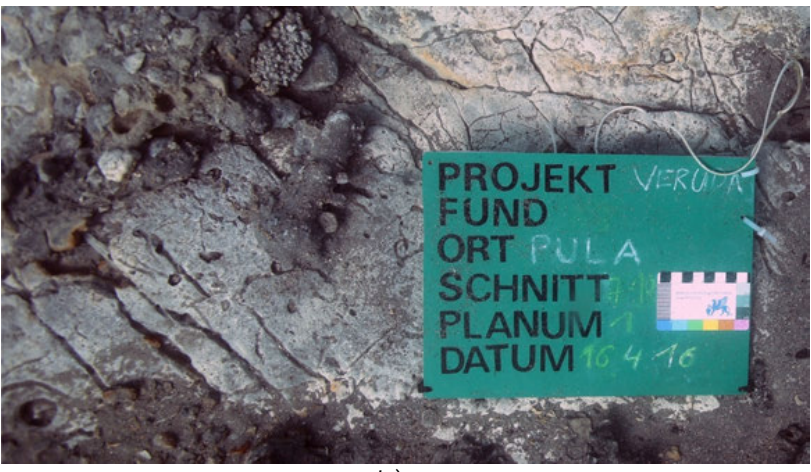

b)

Figure 4. a) an image with a reference color table, taken by Roman Scholz (DAI) with Canon EOS 5D Mark III camera in Veruda/Croatia 2016 (Roman Scholz, DAl); b) right: The improved image using the automatic color correction of Photoshop CS 6.

Photoshop's Auto Color adjusts contrast and color by identifying shadows, midtones, and highlights. The default version neutralizes the midtones using a target color of sRGB 128 gray and clips the shadows and highlight pixels by $0.5 \% 9$. Auto Color is a common method for improving underwater images [Bianco et al. 2015, Bruno et al. 2015].

But, as Fig. 5 shows, this method has its disadvantages.

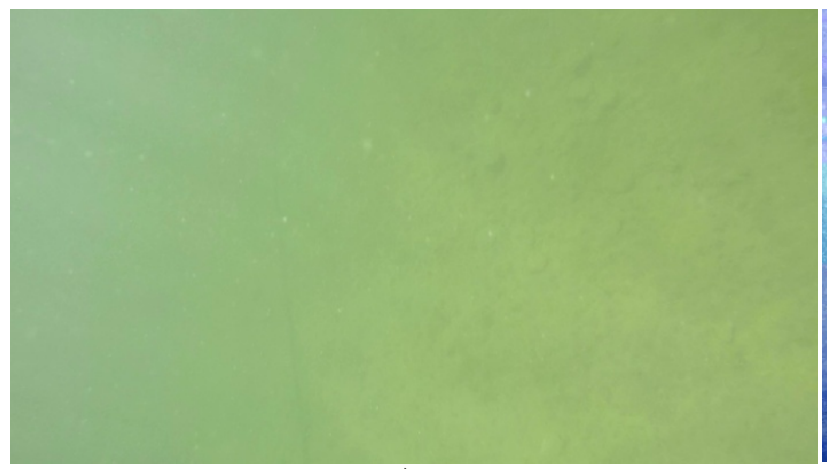

a)

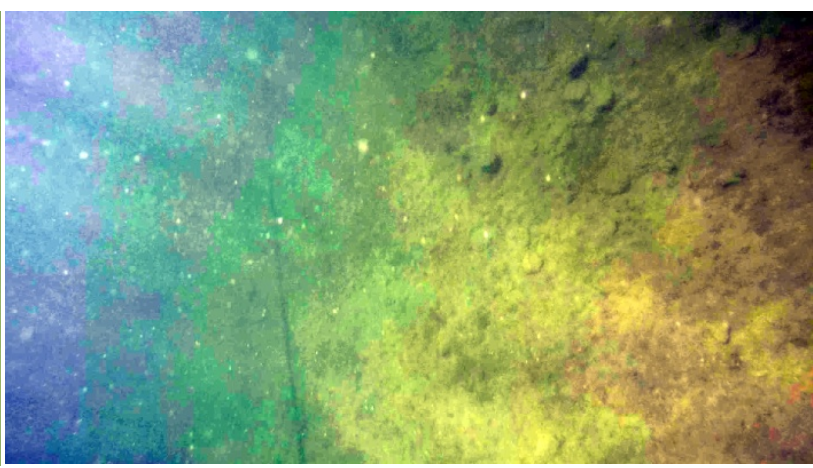

b)

Figure 5. a) an image with low contrast taken with GoPro Hero 4 camera in Mondsee/Austria 2016; b) the improved image using the automatic color correction of Photoshop CS 6.

\footnotetext{
${ }^{9}$ https://helpx.adobe.com/photoshop/using/making-quick-tonal-adjustments.html
} 
To apply this automatic correction to a set of images, it is essential to have similar light conditions and high similarity between the image color spaces. Beside proprietary, paid-for solutions like Photoshop, free programs such as Gimp10 or MS Paint.net1l also feature tools for automatic image enhancement.

During the excavation campaign in Veruda/Croatia in April 2016, this method was applied [Scholz et al. 2016]. The documented shipwreck was excavated, prepared, and documented step by step using a multi-functional support bridge including a measuring slide (Fig. 6).

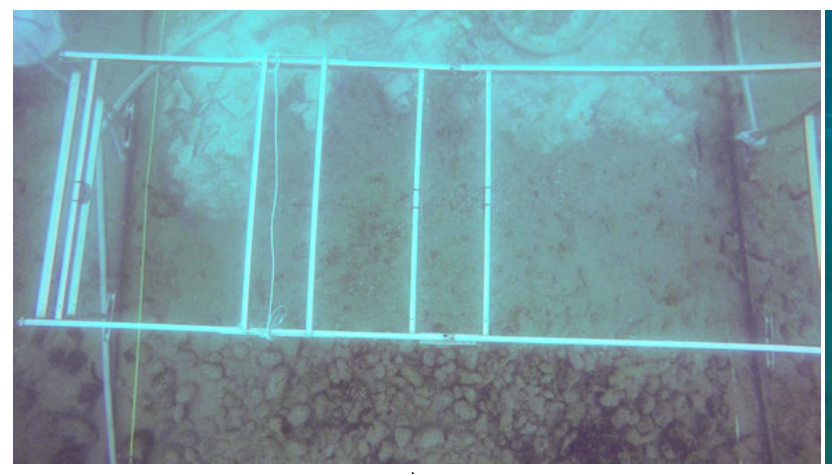

a)

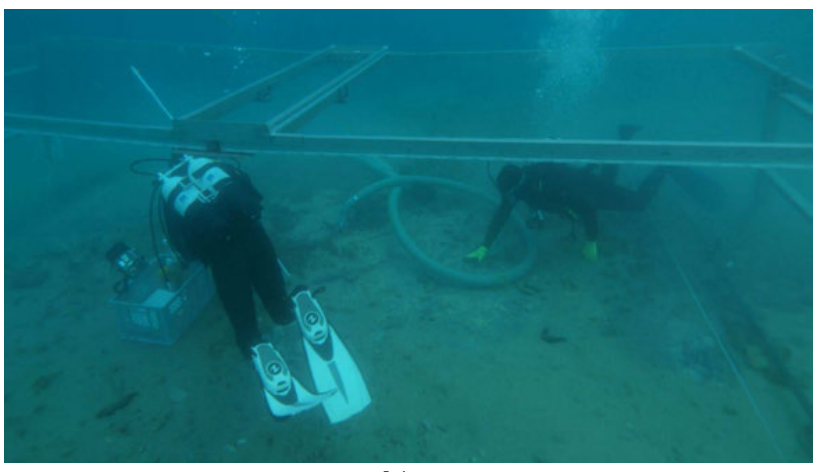

b)

Figure 6. Underwater archaeology often involves the installation of fixed frames. In Veruda a multi-functional support bridge (6x2 meter) including a measuring slide was develop. Photo recorded by: a) Eckbert-ll; b) Mladen Pešić (ICUA) during the excavation process [Scholz et al. 2016].

Afterwards, the challenge was to integrate the recorded images, each taken under different light conditions, into one mosaic using aSPECT3D ${ }^{12}$ (Fig. 7).

To produce this good-looking orthoimage, the team of Roman Scholz required a sophisticated workflow including a multi-functional support bridge to produce images at equal distances and angles [Scholz et al. 2016].

Given the cost and slow speed of operation, it seems more reasonable to search for software solutions that can reliably correct color spectrum differences in input image series.

\footnotetext{
${ }^{10}$ http://www.gimp.org

${ }^{11} \mathrm{http}: / /$ www.getpaint.net

${ }^{12}$ http://aspect.arctron.de/de/
} 


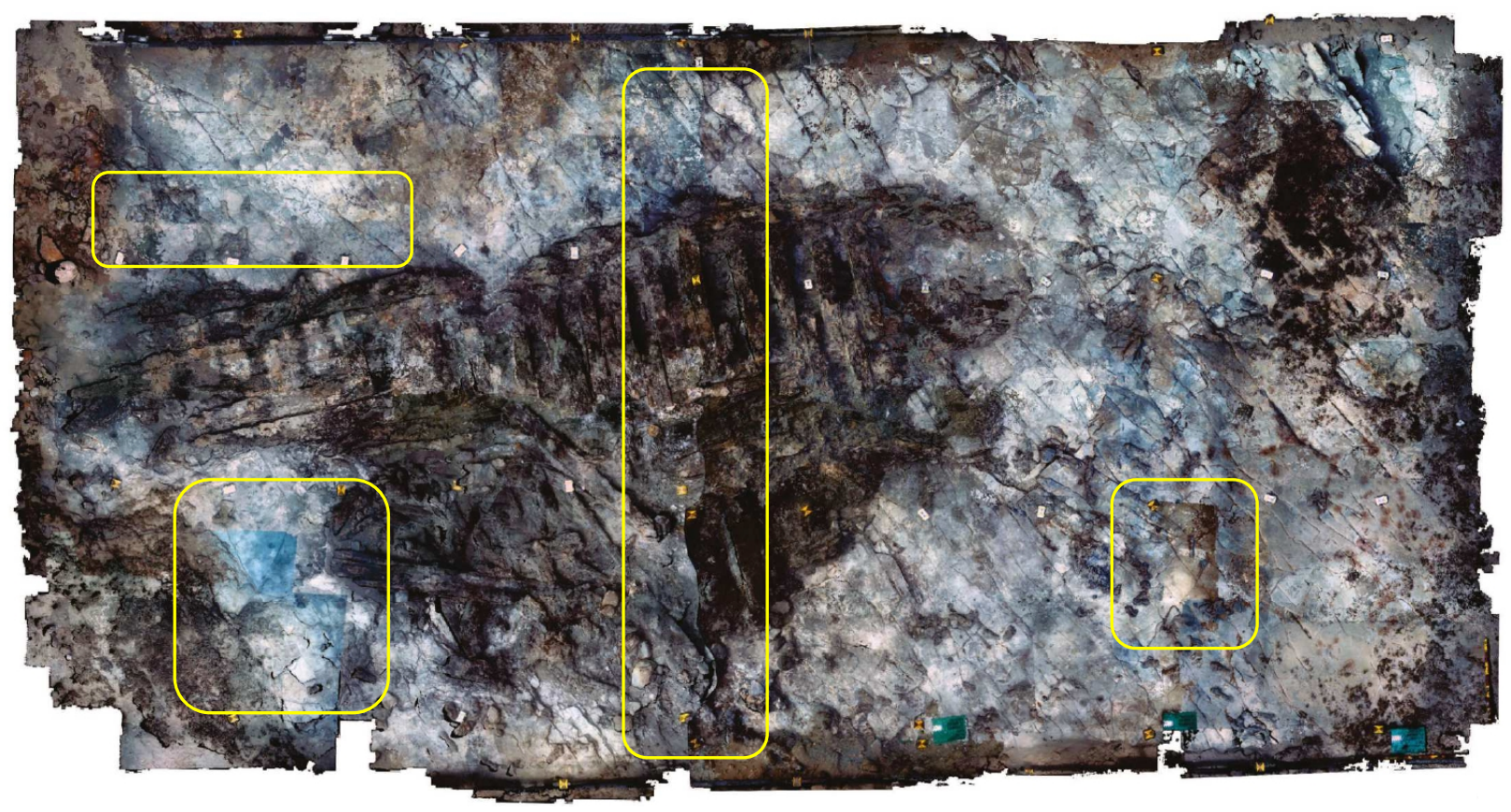

Figure 7. A complete excavation process was photogrammetrically documented by Roman Scholz (DAl) with Canon EOS 5D Mark III camera in Veruda/Croatia 2016 [Scholz et al. 2016]. All photos were corrected by using the automatic color correction of Photoshop CS 6 and a 3d model was reconstructed with aSPECT3D. The resulting orthoimage is shown here. Images with individual color spaces resulting sometimes in blending artefacts (yellow framed boxes).

\subsection{Related image enhancement methods}

Most related approaches of image enhancement for underwater imagery can be classified into image restoration and image enhancement methods [Schettini and Corchs 2010].

Image restoration methods are typically based on optical models which describe the degrading process of the recorded image. Classical linear models [Mertens and Replogle 1977] and other degradation functions like polarization-based methods [Schechner and Karpel 2005] have been examined. In combination with well-known parameters, it is possible to reconstruct the original scenery with near perfection, based on the recorded image. This allows good results, but the determination of the right parameters is often difficult.

Image enhancement approaches do not need any information about the environmental conditions when recording the images. Subjective quality assessment is used to enhance the image data. Often, different classical methods like homomorphic filtering, wavelet denoising, anisotropic filtering and color correction are combined and used independently and successively [Bazeille et al. 2006]. The advantages of different color spaces like HSI [Iqbal et al. 2007] or YCbCr [Bazeille et al. 2006] are also exploited. Due to the subjective factor, the comparison and evaluation of these approaches is more challenging [Schettini and Corchs 2010]. 
The atmospheric haze effect is similar to the effect of image degradation for underwater images [Fattal 2008], but much more dominant. Assuming that we have an image $\vec{J}$ without any haze effects: The longer the distance between observer and surface point, the more the pixel at position $\mathrm{x}$ in the recorded image $\vec{I}$ converges towards the water color $\vec{A}$. The terms "airlight" or "veiling light" are more common for $\vec{A}$ in the research field of haze removal [Koschmieder 1924] and can be used to define the following equation

$$
\vec{I}(x)=t(x) \vec{J}(x)+(1-t(x)) \vec{A}
$$

where $t$ is the mean transmission and can be interpreted as a kind of distance value. The direct influence of $\mathrm{t}$ in $\vec{I}$ as a weight in a linear transformation is shown in Fig. 8.

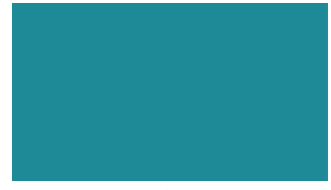

a)

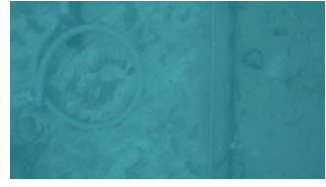

b)

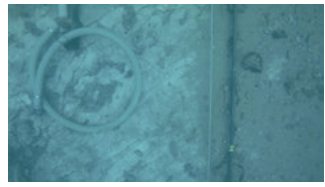

c)

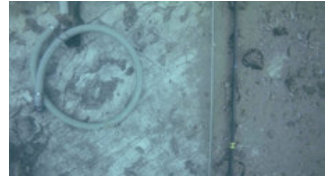

d)

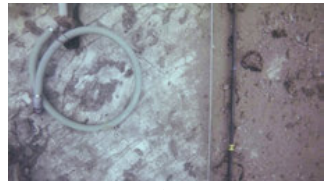

e)

Figure 8. Different values for $t$ and their effects on the image: The image on the left side represents the water color $\vec{A}$ and the image on the right side the image $\vec{J}$ without any haze affects, taken in Veruda/Croatia in 2016 and enhanced: a) $t=0$; b) $t=0.25$; c) $t=0.50$; d) $t=0.75$; e) $t=1.00$.

If $\mathrm{t}=1$ then the distance is zero and we take the real color, if $t=0$ then the distance is infinitive and we take the water color. This equation is defined for all color channels. Haze removal algorithms are focused on restoring the image $\vec{J}$.

This field of research is rich in ideas and touches concepts such as Markov Random Fields (MRF) [Tan 2008] and Independent Component Analysis (ICA) [Fattal 2008]. But the most promising results were produced with the Dark Channel Prior [He et al. 2011] , by which the current version of JEnhancer is also inspired.

\section{AUTOMATIC UNDERWATER IMAGE ENHANCEMENT}

The main idea of the automatic image enhancement process is based on the observation that the existing color spectrum decreases with increasing distance. The best estimate of the water color therefore corresponds to the pixels with the smallest color spectrum. This idea is similar to haze removal approaches using Dark Channel Prior [He et al. 2011]. However, the method discussed here is based on estimating the atmosphere's color, owing to brighter pixels. This is quite different from estimating the water color, which is more green, blue or red/brown.

Research based on Dark Channel Prior often follows rigid approaches, where water is assumed to have most attenuation on the red color channel. Consequently, the dark channel will be estimated by the relation between red and blue/green channels [Carlevaris-Bianco et al. 2010]. Other researchers tried neglecting the red channel [Wen et al. 2013]. 
Our approach, as presented here, is more generic in nature and needs neither a specification of water color nor any other difficult a-priori assumptions. In fact, the only assumption that our automatic methods makes is that there is at least one image in which the natural water color can clearly be seen (see section 4.3).

\title{
3.1 Water color estimation in a single image
}

The color space CIELab is based on the theory of Opponent-process [Hering 1878] and was invented by Commission Internationale de L'Ėclairage (CIE) in 1976 [Commission Internationale de L'Eclairage 1976, Schanda 2007]. Containing all perceivable colors in a perceptual uniform space, it turns the Euclidian distance between two colors to a numeric quantifier for color divergence. Vice versa, applying the same operation on any color results in consistent perceptual changes (Fig. 9).

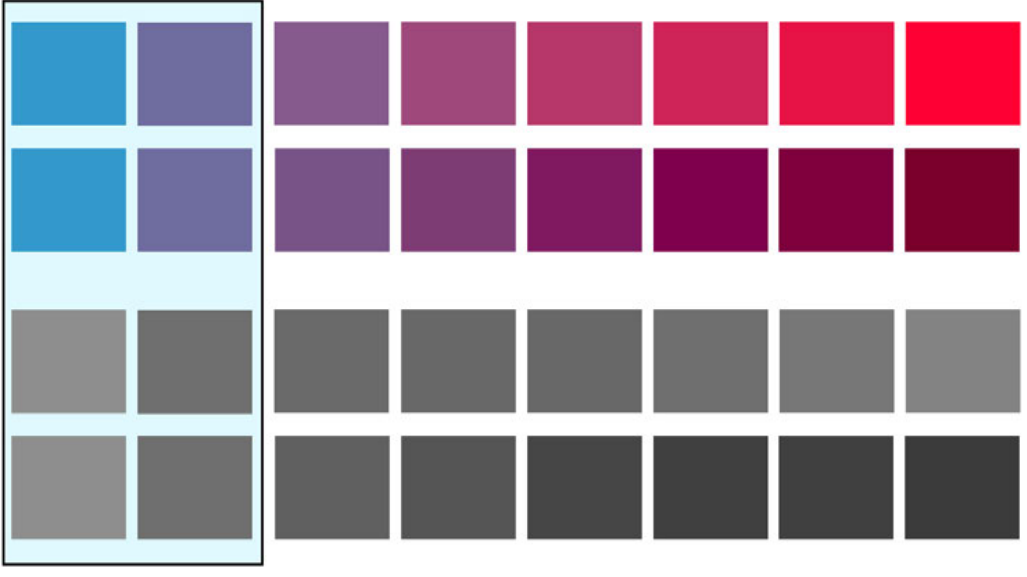

\author{
SRGB \\ CIELab \\ sRGB \\ CIELab
}

Figure 9. The upper group represents the color values of SRGB color space (first row) and CIELab (second row), whereas the lower group shows that correspond with each color's brightness. Colors within the rectangle on the left side are identical, while all other colors are interpolated in equal steps from the light blue to the right end color. As shown above, the SRGB colors are first getting darker, followed by an increasing brightness, while the CIELab colors are continuously decreasing in brightness.

Our motivation for choosing CIELab as color space instead of e.g. sRGB will become clear with the last step discussed in section 3.4, when color correction is applied.

Furthermore, colors are expressed as degrees, using the polar coordinates $a$ and $b$ and their luminance L. Therefore, colors are independent from primary color channels like sRGB and more general.

All images will be converted to a modified CIELab space, representing a unit ball. To estimate the water color of an image $I$ given in CIELab color space, we detect the local minimum and maximum for every pixel $X$ in a neighborhood $\Omega(X)$ around the center $X$ separately for every color channel C. The size of the neighborhood $\Omega(X)$ depends strongly on the image content. As an interim result, we calculate the intensity maps $I_{\min }^{C}(X)$ and $I_{\max }^{C}(X)$ : 


$$
I_{\min }^{C}(X)=\min _{Y \in \Omega(X)}\left(I^{C}(Y)\right) \text { and } I_{\max }^{C}(X)=\max _{Y \in \Omega(X)}\left(I^{C}(Y)\right)
$$

One color channel of image $I$ with a high variance

$$
I_{\text {var }}^{C}(X)=\left(I_{\text {max }}^{C}(X)-I_{\text {min }}^{C}(X)\right)
$$

is sufficient to exclude the pixel $X$ from of the list of candidates. Therefore, we detect the maximum of all color channels:

$$
I_{v a r}(X)=\max _{i \in C}\left(I_{v a r}^{i}(X)\right)
$$

Inside this map of variance, the position of the minimum represents the area with the smallest color spectrum all over the image and is therefore a high potential candidate to represent the water color.

\subsection{Extension to pyramid approach}

Plain, uni-colored areas can still be a problem. To minimize their impact, an image pyramid $\hat{I}$ of the image $\vec{I}$ is first calculated. The water color estimation is now applied on every scale, which results in:

$$
I_{\text {var }_{\text {final }}}(X)=\sum_{k=0}^{s} I_{\text {var }_{2-k}}(X)
$$

The minimum represents the water color, so we just need to detect the right position:

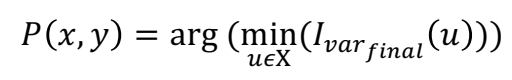

Finally, to reduce up and down scaling while estimating the water color out of a sequence of images, a mean value can be applied

$$
A_{n}=\frac{n-1}{n} A_{n-1}+\frac{1}{n} I(P)
$$

where $I(P)$ represents the position of the estimated water color in image $\vec{I}$.

\subsection{Water color estimation algorithm}

To detect the water color $\vec{A}$ of an image set $\vec{I}_{1}, \vec{I}_{2}, \ldots, \vec{I}_{1 n}$, the following algorithm, given in pseudocode, is applied:

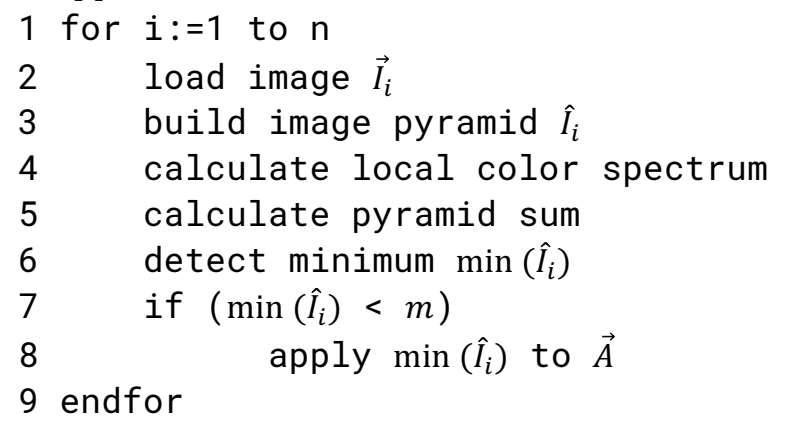


The bias $m$ (see line 7 of the pseudocode above) represents the contrast threshold and is currently set to $6 \%$, which is three times higher than the contrast threshold of $2 \%$ visible to human beings [Koschmieder 1924]. Underwater images taken at low light conditions are prone to noise. To reduce the influence of noise, we empirically chose a higher setting for $m$.

\subsection{Correction of color space}

We use the advantage of CIELab now: The exteriors represent fully saturated colors and these colors are free of distortion effects, such as those caused by absorption.

Transforming Koschmieder's equation to vector representation, makes it easier to understand the reasoning behind the color manipulation:

$$
\vec{I}(x)=\vec{A}+t(x)(\vec{J}(x)-\vec{A})
$$

The color of each given image pixel has its origin in the water color $\vec{A}$. The vector direction is given by the difference between original color $\vec{J}(x)$ and $\vec{A}$ using the transmission $t$ as parameter within the range $t \in[0,1]$. In this representation, it is obvious that every color has to be on a line between the origin color and the water color $\vec{A}$. Due to the fact that JEnhancer works without any depth maps, the origin color has to be at least the given color pixel with distance $=0$, following the line up to the border of the color space.

Furthermore, it is ensured for border colors to be free of any possible manipulation. JEnhancer tries to restore these pure colors. In fact, He's Dark Channel Prior follows a similar approach to the lower border in SRGB, by restoring colors to achieve a zero value in at least one of its channels [He et al. 2011]. Due to the modification of CIELab, by representing a unit ball, every single border color has a radius $=1$ with:

$$
|\vec{J}|=\sqrt{J_{L}^{2}+J_{a}^{2}+J_{b}^{2}}=1
$$

With this information, it is possible to transform Koschmieder's equation towards an alternate ball equation

$$
t^{2}=\left(I_{L}-(1-t) A_{L}\right)^{2}+\left(I_{a}-(1-t) A_{a}\right)^{2}+\left(I_{b}-(1-t) A_{b}\right)^{2}
$$

in which the transmission $t$ is the only unknown variable. Therefore, the problem is solvable by reducing it to a quadratic equation:

$$
t^{2}\left(\left(\vec{A} \vec{A}^{T}\right)-1\right)+2 t\left(\left(\vec{I} \vec{A}^{T}\right)-\left(\vec{A} \vec{A}^{T}\right)\right)+\left((\vec{I}-\vec{A})(\vec{A}-\vec{A})^{T}\right)=0
$$

Finally, using the well-known "abc formula" determinates, the value of $t$, where $t$ has to be a positive value:

$$
a t^{2}+2 b t+c=0 \rightarrow t_{1,2}=\frac{-b \pm \sqrt{b^{2}-a c}}{a}
$$

As this calculated transmission will cause every color to settle at the CIELab border, the transmission $t$ has to be refined. Otherwise the resulting image will have over-saturated colors. The current 


\section{1:578 M. Block et al.}

implementation of JEnhancer takes a power smaller than one from $t$, to fulfill a higher alternation to median values and almost no change to values near $t=0$ and $t=1$. Actually, this power is set to 0.95 , but can be adjusted to fulfill application-based needs. An alternative way of refinement is possible as long as the transmission gets a higher value than calculated.

Afterwards, the restoring process firstly subtracts the influence of the estimated water color which results in the weakened origin image $J^{\prime}$ :

$$
t \vec{J}=\vec{I}-(1-t) \vec{A}=J^{1}
$$

Remembering the nature of CIELab, the weakened image has at this moment its characteristic colors, because the division of $t$, according to $\vec{J}=J^{l} / t$, only alters the color's luminance and saturation, but not the degree. Therefore, this step enhances the contrast of the final image. Once more the transmission has to be refined, due to the fact that the sRGB gamut is a sub space within CIELab and the regular output will use sRGB, e.g. JPEG. The current implementation of JEnhancer uses the third root of $t$, but as above, different solutions are possible to adjust the transmission for the contrast enhancement step. To show the two major steps of the enhancement process, three examples are shown in Figs. 15-17 (see Appendix).

Our image enhancement method has been implemented in the software JEnhancer which we provide free for research purposes. The current version of the software, along with installation and usage instructions, is available from our project page $\mathrm{e}^{13}$.

\section{EXPERIMENTS AND INTERIM RESULTS}

JEnhancer was successfully used in several campaigns in Kamenz/Germany, Zwischenahner Meer/Germany, Veruda/Croatia [Scholz et al. 2016] and Mondsee/Austria [Block et al. 2016, Pile Dwellings], since February 2016. JEnhancer is not only used to improve images in the sense of color restoration, it is also used to improve an image, so that a feature detector will be able to detect more significant features for SfM-based 3d reconstruction.

Out of the past campaigns and experience gained, we first want to show the influence JEnhancer has on the input images. After that, we want to compare some suitable image sets and the different outcomes of Photoshop and JEnhancer, and finally show some unsuitable image sets.

\subsection{Feature enrichment experiment}

The method we have presented here has the advantages of improving underwater images automatically and of involving all images in the process. Beside the color correction for the overall $3 \mathrm{~d}$ model, one aim of the image enhancement process is to increase the potential number of detected features for SfM reconstruction. When using small images with blurry content, feature detection often fails or detects insufficient numbers of features, so that image correlations cannot be found.

To compare the outcomes of Photoshop and JEnhancer according to the number of detected features and the produced $3 \mathrm{~d}$ models, a challenging image set of the campaign in Mondsee/Austria was

\footnotetext{
${ }^{13}$ http://www.archaeonautic.de
} 
selected. The videos of three parallel cameras starting with a camera to bottom distance of about 3 meters and and increasing to a distance of up to six meters were used. The images at the end of the videos are too blurry to detect any serious data for the $3 \mathrm{~d}$ reconstruction process. The challenge is now to combine as many images as possible in the pose estimation step.

As an experimental image set, we used 1560 images, extracted from a GoPro Hero 4 BE video with a resolution of about 1980×1080 pixels, taken at Mondsee/Austria in 2016 [Block et al. 2016, Pile Dwellings]. To optimize the output of VisualSFM ${ }^{14}$, we reduced the image size to $1600 \times 900$ pixels. This set M1 represents the original data set Then, set M2 represents the results of Photoshop's automatic color correction and M3 the results of JEnhancer. To all sets M1, M2, and M3, SiftGPU [Wu 2007] with pre-trained parameters was applied. The images are sorted by number of features found in M1. To give an idea about image quality, three different examples are selected (Fig. 10).
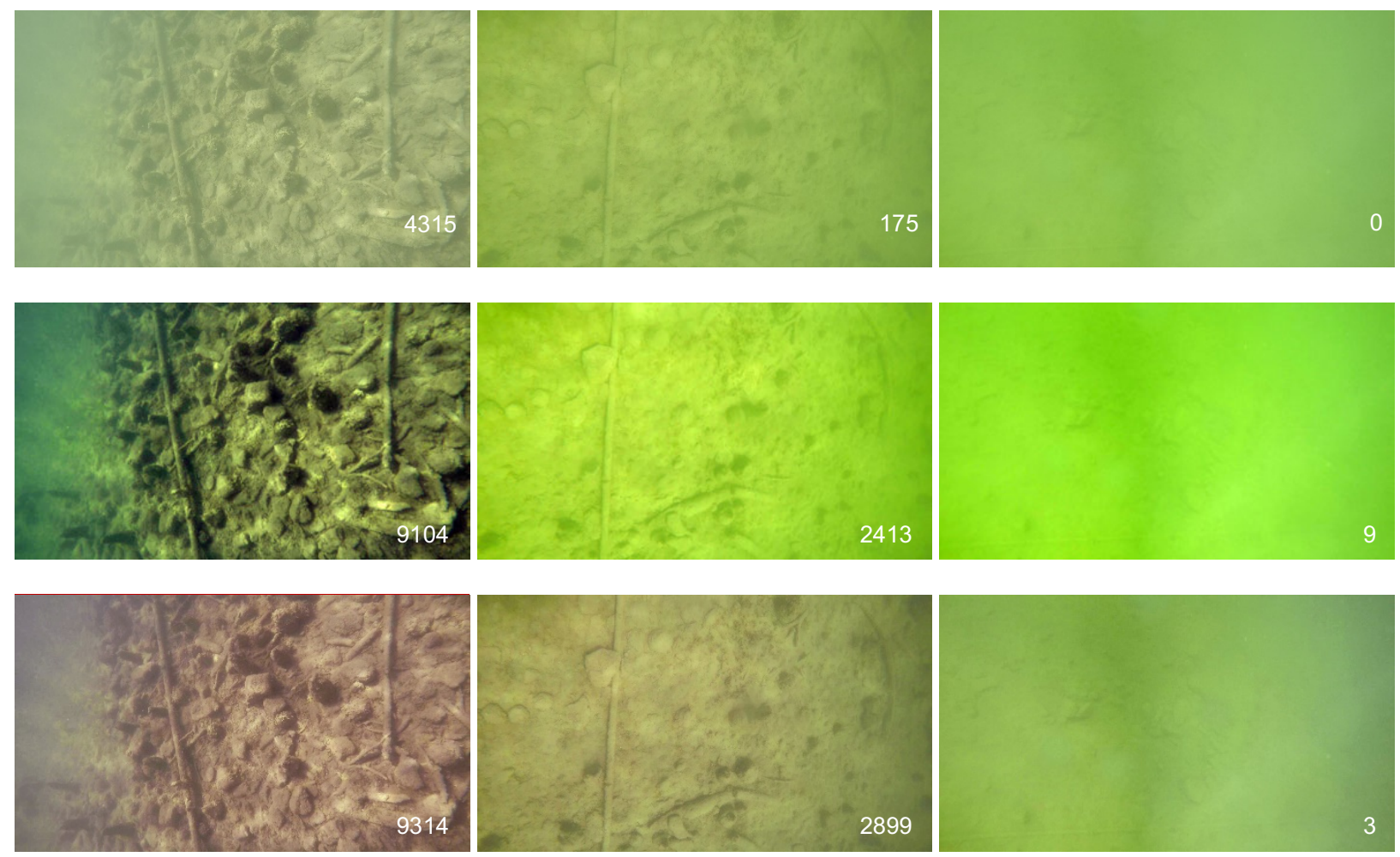

Figure 10. The top row shows the original data, taken by the GoPro Hero 4 BE with 1080p, and the second row shows the same images enhanced with Photoshop's automatic color correction. The third row shows the results of JEnhancer. The number of detected features with SiftGPU is shown inside every image. The first column shows the best image out of the 1560 images. The second column shows an intermediate image out of the middle of the data set. The third and last column shows the worst image.

\footnotetext{
${ }^{14}$ http://ccwu.me/vsfm/doc.html
} 


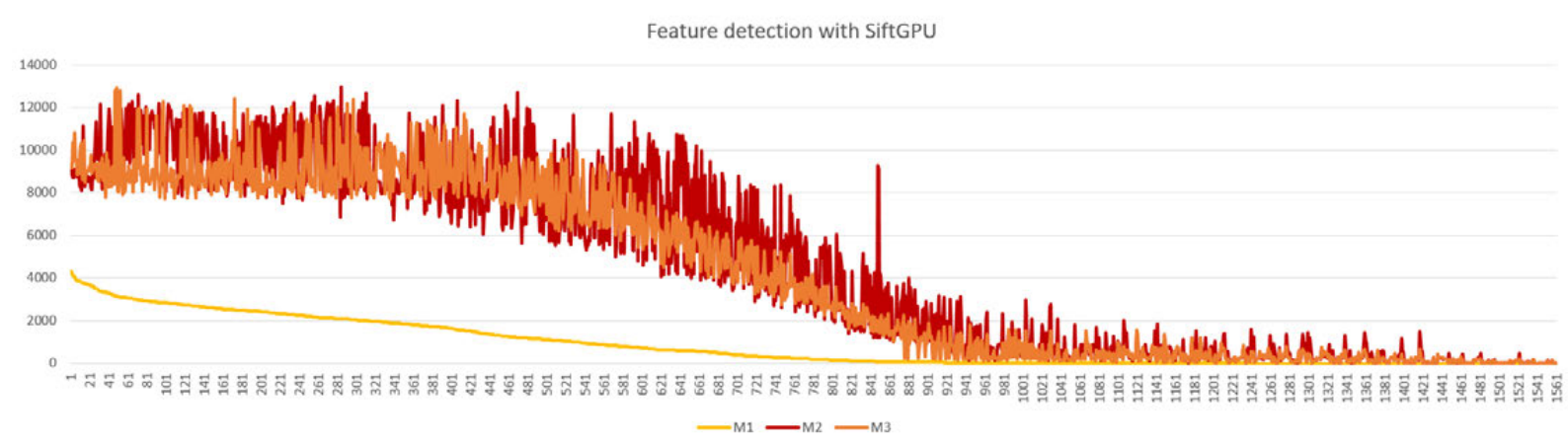

Figure 11. All 1560 images are plotted along the $x$ (horizontal) axis. The yellow graph shows the number of features, found by SiftGPU with pretrained parameters in the original data set M1. The red graph shows the features detected in the improved version of the images in set M2 using Photoshop and the orange graph the number of features detected using JEnhancer. The sum of all features in $M 1$ is 1333287, in M2 7276192, and in M3 6632938. The mean value in M1 is around 855 features per image, in M2 around 4664 and in M3 around 4251.

The diagram in Fig. 11 shows the number of features extracted from the original data (yellow plot), the images improved by Photoshop (red plot), and the image data improved by JEnhancer (orange plot).

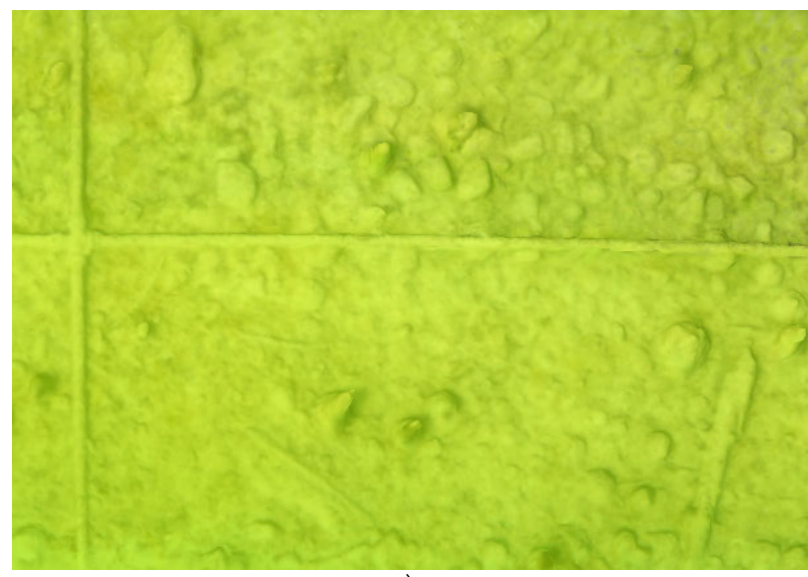

a)

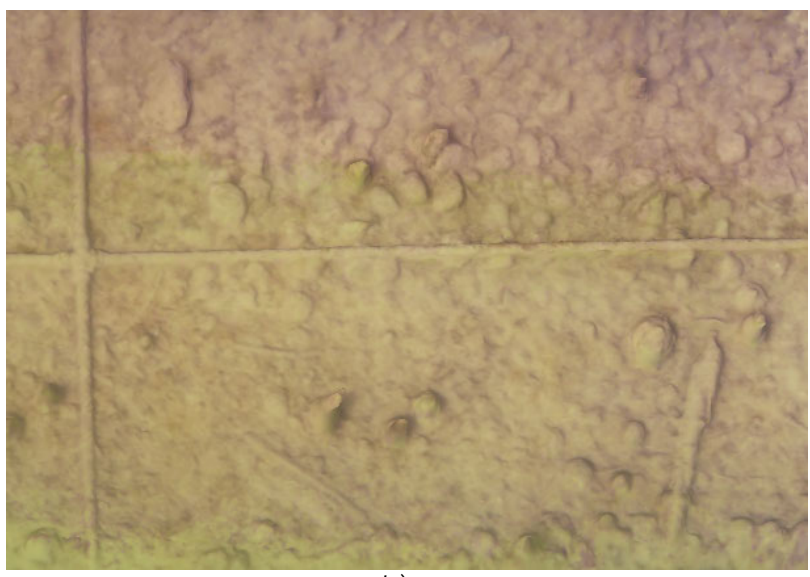

b)

Figure 12. One selected part of the resulting 3d model using CMPMVS where results of Photoshop (a) and results of JEnhancer (b) were used. JEnhancer is closer to the muddy underground situation and produces more realistic results.

Thus, the sum of features detected in all images using JEnhancer was amplified by a factor of 5 and via Photoshop by a factor of 5.4. A sufficient number of features is important to find the right correlations between images. The pose estimation using VisualSFM delivers for the original image data set M1 one connected model (50659 3d points) with 350 images, for M2 one model (238542 3d points) with 528 images and for M3 one model (187041 3d points) with 514 images. On the one hand, both Photoshop and JEnhancer are able to improve images to identify more points and finally more 
correlations. On the other hand, the quality of the resulting $3 \mathrm{~d}$ models depends strongly on the quality of the input images.

If lighting and seen color space are more or less equal, Photoshop's results are quite useful (as seen in Section 2.4). These properties are present in some parts of the selected image set. The main difference between Photoshop and JEnhancer is the transformed color space. Whereas Photoshop only samples the original colors to enhance the color space and increases the contrast in comparison to the original images, JEnhancer analyses the water color and eliminates it, as seen in Fig. 12.

The main difference, between Photoshop and JEnhancer takes effect when lighting and seen color space are diverging. Here, we produce visual artefacts and inconsistences in parts of the $3 \mathrm{~d}$ model as seen in Fig. 13.

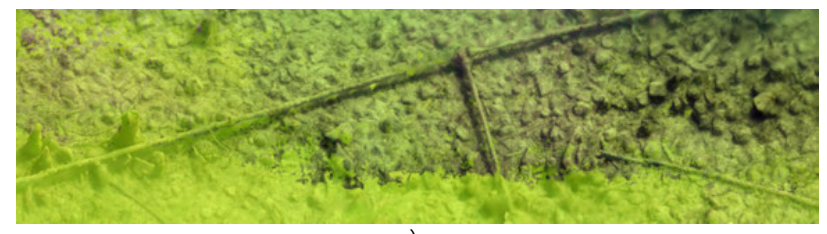

a)

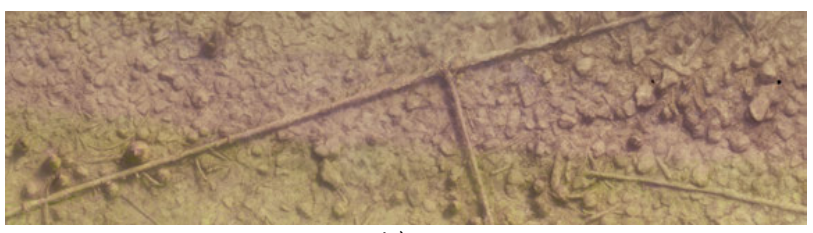

b)

Figure 13. a) improving images independently (Photoshop) sometimes may lead to visual artefacts, when differences in lighting and seen color space occurs; b) JEnhancer produces results with homogenous color spectrum.

The results indicate, that Photoshop's outcome can be used to find more correlations between images, and later JEnhancer's outcome can be used to better texturize the model.

\subsection{Examples of suitable image sets}

Since February 2016, JEnhancer is an essential component of the 3d reconstruction pipeline Archaeo3D. To compare the outputs of JEnhancer and Photoshop, Fig. 15 (see Appendix) shows some suitable examples.

Whereas Photoshop only improves the histograms and therefore increases the contrast and hue, JEnhancer corrects the image according to the estimated water color. Both methods increase the number of detected features in most cases, because feature extraction depends strongly on image sharpness and structure.

\subsection{Examples of unsuitable image sets}

As discussed in Section 3.2, not all scenarios or image sets are suitable for enhancement by JEnhancer. If the assumption of an unobstructed field of view to detect the water color does not hold, the quality of the resulting images will be poor, with wrongly compensated colors (Fig. 14). 

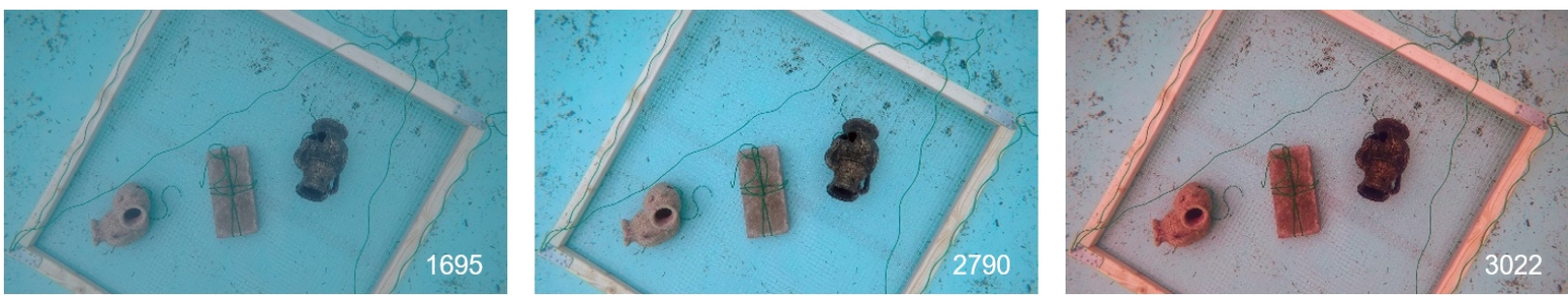

Figure 14. This image was taken in a pool in Radebeul/Germany. The assumption of the presence of the water color fails here. The result of Photoshop in the middle is close to the real scene while JEnhancer estimates blue as the water color and corrects the axis in a way that turns the water color to gray.

If JEnhancer is used to improve an image set, it is recommending taking some images with an unobstructed field of view. This is important to be able to fulfill the assumption that the water color is clearly seen in a minimum of one image.

\section{CONCLUSIONS AND FUTURE WORK}

This paper presented the first freely available (for research purposes) software tool for automatic enhancement of underwater image sets using Improved Dark Channel Prior. Experiments have shown that JEnhancer can significantly increase the number of detected features in an SfM processing chain, which is of crucial importance for constructing complete $3 \mathrm{~d}$ models when extremely blurry images are used. In one experiment with 1560 images, the sum of features was amplified by a factor of 5 in comparison to non-enhanced images using JEnhancer and by factor 5.4 using Photoshop. The results indicate, that Photoshop's outcome can be used to find more correlations between images, and later JEnhancer's outcome can be used to better texturize the model.

The module JEnhancer is one more useful piece in the 3d reconstruction pipeline Archaeo3D. The decision to offer it as free software for research purposes, is in alignment with the project's philosophy. After a software refactoring step, we plan to release it as an open source project to invite more people to work on it. Beside improving the software, we are collecting different image sets for benchmarking and developing an automatic evaluation process for comparing different approaches.

\section{ACKNOWLEDGEMENTS}

The excavation campaign in Veruda/Croatia 2016 was financially supported by the Frost Foundation. The project Archaeonautic is funded by the Saxon State Ministry for Science and the Arts. Furthermore, we would like to thank all the people who supported our team in various ways.

\section{REFERENCES}

C. Balletti et al. 2015. Underwater Photogrammetry and 3D Reconstruction of Marble Cargos Shipwreck. In The International Archives of the Photogrammetry, Remote Sensing and Spatial 
Information Sciences XL-5/W5 (2015), 7-13.

Herbert Bay et al. 2008. Speeded-up Robust Features (SURF). Computer Vision and Image Understanding (CVIU) 110, 3 (2008), 346-359.

Stéphane Bazeille et al. 2006. Automatic Underwater Image Pre-processing. In Proceedings of the Caracterisation du Milieu Marin (CMM '06).

Nicholas Carlevaris-Bianco, Anush Mohan, and Ryan M. Eustice. 2010. Initial Results in Underwater Single Image Dehazing. In Proceedings of the IEEE/MTS OCEANS Conference and Exbibition, Seattle, WA, USA, September 2010, 1-8.

Juli Berwald et al. 1995. Influences of Absorption and Scattering on Vertical Changes in the Average Cosine of the Underwater Light Field. Journal of Limnology and Oceanography 40, 8 (1995), 1347-1357.

Gianfranco Bianco et al. 2015. A New Color Correction Method for Underwater Imaging. In The International Archives of the Photogrammetry, Remote Sensing and Spatial Information Sciences XL-5/W5 (2015), 25-32.

Marco Block et al. 2016. Eckbert-II: Mini-U-Boot zur Sondierung und Dokumentation von archäologischen Fundstellen. Technical Report. DD-2016-01, FITS, HTW Dresden, Germany.

Marco Block, et al. 2016. Underwater Videogrammetry with Adaptive Feature Detection at "See am Mondsee" , Austria. Studies in Digital Heritage 1 (2017).

Fabio Bruno et al. 2015. 3d Documentation of Archeological Remains in the Underwater Park of Baiae. In The International Archives of the Photogrammetry, Remote Sensing and Spatial Information Sciences XL-5/W5 (2015), 41-46.

Commission Internationale de L'Eclairage. 1976. CIE Colorimetry - Part 4: 1976 L*a*b* Colour Space. ISO 11664-4:2008(E).

Fabio Cozman and Eric Krotkov. 1997. Depth from Scattering. In IEEE Conference on Computer Vision and Pattern Recognition (CVPR) 31 (1997), 801-806.

Marc Pierrot Deseilligny and Isabelle Cléry. 2011. Apero, an Open Source Bundle Adjustment Software for Automatic Calibration and Orientation of Set of Images. In ISPRS - International Archives of the Photogrammetry, Remote Sensing and Spatial Information Sciences, Volume XXXVIII-5/W16 (2011), 269-276

Raanan Fattal. 2008. Single Image Dehazing. In ACM Transactions on Graphics (TOG) 27, 3 (2008), 72:1-72:9.

Benjamin Gehmlich and Marco Block. 2015. Diversity of Flight Strategies in UAV Recording. In 20th Conference on Cultural Heritage and New Technologies, Vienna, Austria.

John A. Greenwood. 1999. Large Component Deformation Studies Using Videogrammetry. In The 6th International Workshop on Accelerator Alignment, Grenoble, France (IWAA) 32, 50 (1999), 133.

Richard Hartley and Andrew Zisserman. 2004. Multiple View Geometry in Computer Vision. Second edition. Cambridge: Cambridge University Press.

Kaiming He et al. 2011. Single Image Haze Removal Using Dark Channel Prior. In IEEE Transactions on Pattern Analysis and Machine Intelligence 33, 12 (2011), 2341-2353.

Ewald Hering. 1878. Zur Lehre vom Lichtsinne - Sechs Mittheilungen an die Kaiserl. Akademie der Wissenschaften in Wien. Zweiter, unveränderter Abdruck. Gerold. Wien.

Kashif Iqbal et al. 2007. Underwater Image Enhancement Using an Integrated Colour Model. In IAENG International Journal of Computer Science (IJCS) 34, 2 (2007), 529-534. 


\section{1:584 M. Block et al.}

Kashif Iqbal et al. 2010. Enhancing The Low Quality Images Using Unsupervised Colour Correction Method. In IEEE International Conference on Systems Man and Cybernetics (SMC), (2010), 17031709.

Jules S. Jaffe et al. 2001. Underwater Optical Imaging: Status and Prospects. Oceanography 14, 3 (2001), 64-75.

Michal Jancosek and Tomas Pajdla. 2011. Multi-View Reconstruction Preserving Weakly-Supported Surfaces. In IEEE Conference on Computer Vision and Pattern Recognition (CVPR) Vol. \#? (2011), 3121-3128.

Harald Koschmieder. 1924. Theorie der horizontalen Sichtweite: Kontrast und Sichtweite, Beiträge zur Physik der freien Atmosphäre (1924), 33-53 and 171-181.

Hyon Lim et al. 2014. Online 3D Reconstruction and 6-DoF Pose Estimation for RGB-D Sensors. In 5th Workshop at the European Conference on Computer Vision. Zurich, Switzerland (2014), 238254.

David G. Lowe. 1999. Object Recognition from Local Scale-invariant Keypoints. In IEEE International Conference on Computer Vision (ICCV) 99, 2 (1999), 1150-1157.

Huimin Lu et al. 2013. Underwater Image Enhancement Using Guided Trigonometric Bilateral Filter and Fast Automatic Color Correction. In 20th IEEE International Conference on Image Processing (ICIP) (2013), 3412-3416.

Lena E. Mertens and Frank S. Replogle. 1977. Use of Point Spread and Beam Spread Functions for Analysis of Imaging Systems in Water. Journal of the Optical Society of America 67, 8 (1977), $1105-1117$.

Ondrej Miksik and Krystian Mikolajczik. 2012. Evaluation of Local Detectors and Descriptors for Fast Feature Matching. In 21st International Conference on Pattern Recognition (ICPR) (2012), 2681-2685.

Curtis D. Mobley. 2001. Radiative Transfer in the Ocean. In Encyclopedia of Ocean Sciences. Cambridge, MA, Academic Press 2001, 2321-2330.

Emmanuel Moisan et al. 2015. Building a 3D Reference Model for Canal Tunnel Surveying Using Sonar and Laser Scanning. In The International Archives of the Photogrammetry, Remote Sensing and Spatial Information Sciences, XL-5/W5 (2015), 153-159.

Pierre Moulon et al. 2013. Global Fusion of Relative Motions for Robust, Accurate and Scalable Structure from Motion. In IEEE International Conference on Computer Vision (ICCV) (2013), 3248-3255.

David Nistér. 2004. Automatic Passive Recovery of 3D from Images and Video. In Proceedings of The Second International Symposium on 3D Data Processing, Visualization \& Transmission (3DPVT04). Thessaloniko, Greece, (2004), 438-445.

Kimon Papadimitriou. 2015. Course Outline for a Scuba Diving Speciality "Underwater Survey Diver." In The International Archives of the Photogrammetry, Remote Sensing and Spatial Information Sciences XL-5/W5K (2015), 161-166.

Richard S. Pappa et al. 2003. Dot-Projection Photogrammetry and Videogrammetry of Gossamer Space Structures. Journal of Spacecraft and Rockets 40, No. 6 (2003), 858-867.

Marc Pollefeys et al. 2004. Visual modeling with a hand-held camera. In International Journal of Computer Vision 59, 3 (2004), 207-232.

Elisa Pruno et al. 2015. Underwater Photogrammetry Methods for a Peculiar Case-Study: San 
Domenico (Prato, Italy). In The International Archives of the Photogrammetry, Remote Sensing and Spatial Information Sciences XL-5/W5 (2015), 171-176.

Fabio Remondino. 2011. Heritage Recording and 3D Modeling with Photogrammetry and 3D Scanning. Remote Sensing 3, 6 (2011), 1104-1138.

Pedro Santos and Martin Ritz. 2013. CultLab3D - Fast and Economic, High Quality 3D Digitization of Cultural Heritage Artifacts. Photogrametric Week '13, 29. Stuttgart, Germany.

János Schanda. 2007. Colorimetry: Understanding the CIE system. Hoboken, New Jersey, John Wiley \& sons.

Yoav Y. Schechner and Nir Karpel. 2005. Recovery of Underwater Visibility and Structure by Polarization Analysis. IEEE Journal of Oceanic Engineering 30, 3 (2005), 570-587.

Raimondo Schettini and Silvia Corchs. 2010. Underwater Image Processing: State of the Art of Restoration and Image Enhancement Methods. In EURASIP Journal on Advances in Signal Processing (2010), 1-14.

Roman Scholz et al. 2016. Shipwreck Documentation in Veruda Using Photo- and Videogrammetry. In 21st Conference on Cultural Heritage and New Technologies (CHNT 21), Vienna, Austria.

Noah Snavely et al. 2006. Photo Tourism: Exploring Photo Collections in 3D. In ACM transactions on graphics (TOG) 25, 3 (2006), 835-846.

Dariusz Stramski et al. 2004. The Role of Seawater Constituents in Light Backscattering in the Ocean. Progress in Oceanography 61, 1 (2004), 27-56.

Robby T. Tan. 2008. Visibility in Bad Weather from a Single Image. In IEEE Computer Society Conference on Computer Vision and Pattern Recognition (CVPR) (2008), 1-8.

Salvatore Troisi et al. 2015. 3D Models Comparison of Complex Shell in Underwater and Dry Environments. The International Archives of the Photogrammetry, Remote Sensing and Spatial Information Sciences XL-5/W5 (2015), 215-222.

Ying-mei Wei et al. 2013. Applications of Structure from Motion: A Survey. Journal of Zhejiang University 14, 7 (2013), 486-494.

Haocheng Wen et al. 2013. Single Underwater Image Enhancement with a New Optical Model. In IEEE International Symposium on Circuits and Systems (ISCAS) (2013), 753-756.

Changchang Wu. 2007. SIFTGPU: A GPU Implementation of Scale Invariant Feature Transform. http://www.cs.unc.edu/ ccwu/siftgpu/ 


\section{APPENDIX}
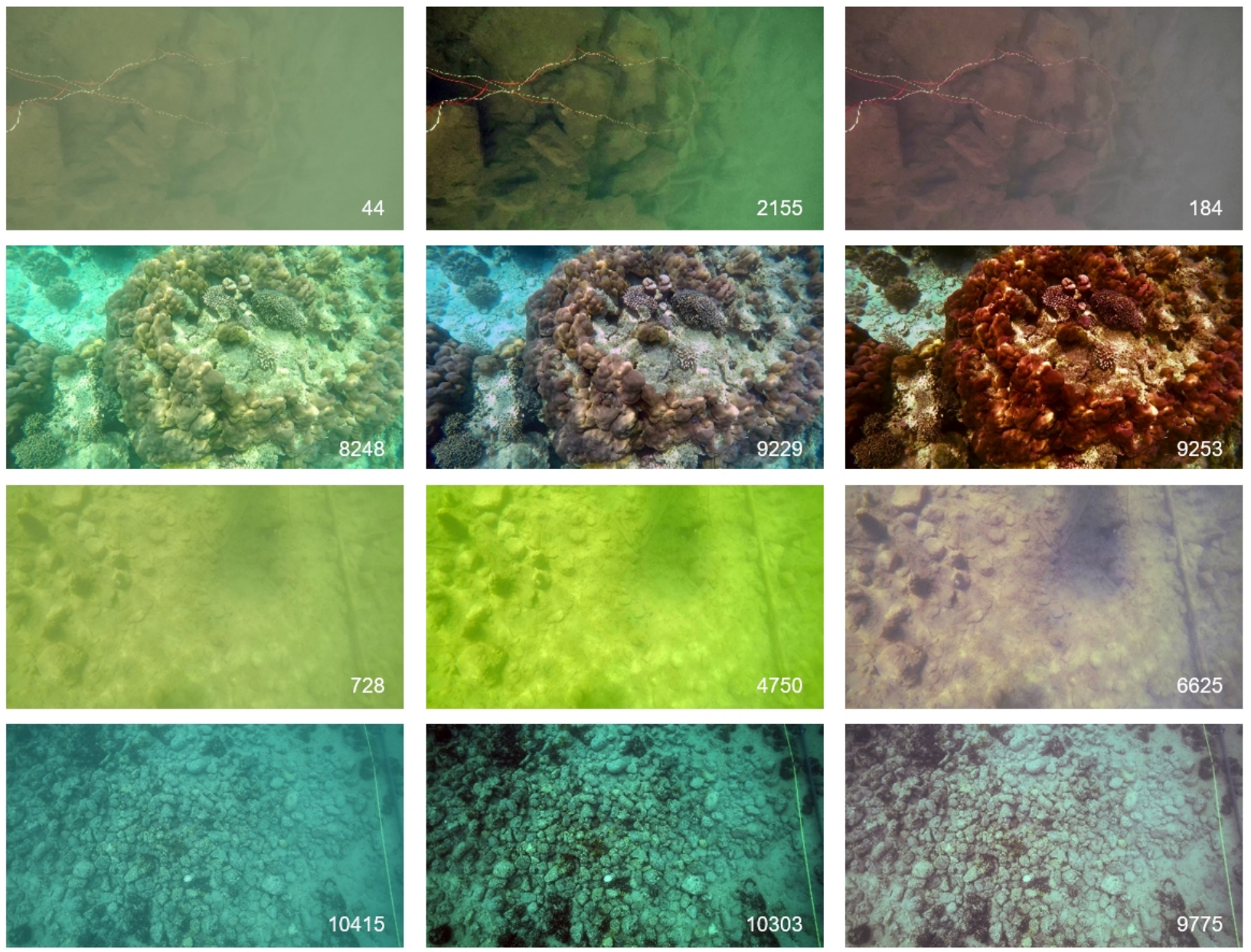

Figure 15. The number of detected features (using SiftGPU) is shown inside every image. The first column shows four selected examples of Kamenz/Germany, Surin Islands/Thailand, Mondsee/Austria and Veruda/Croatia, beginning at the top. The second column shows the results of the automatic image enhancement using Photoshop and the third the results of JEnhancer. At the bottom of every image, the count of detected features is shown. 

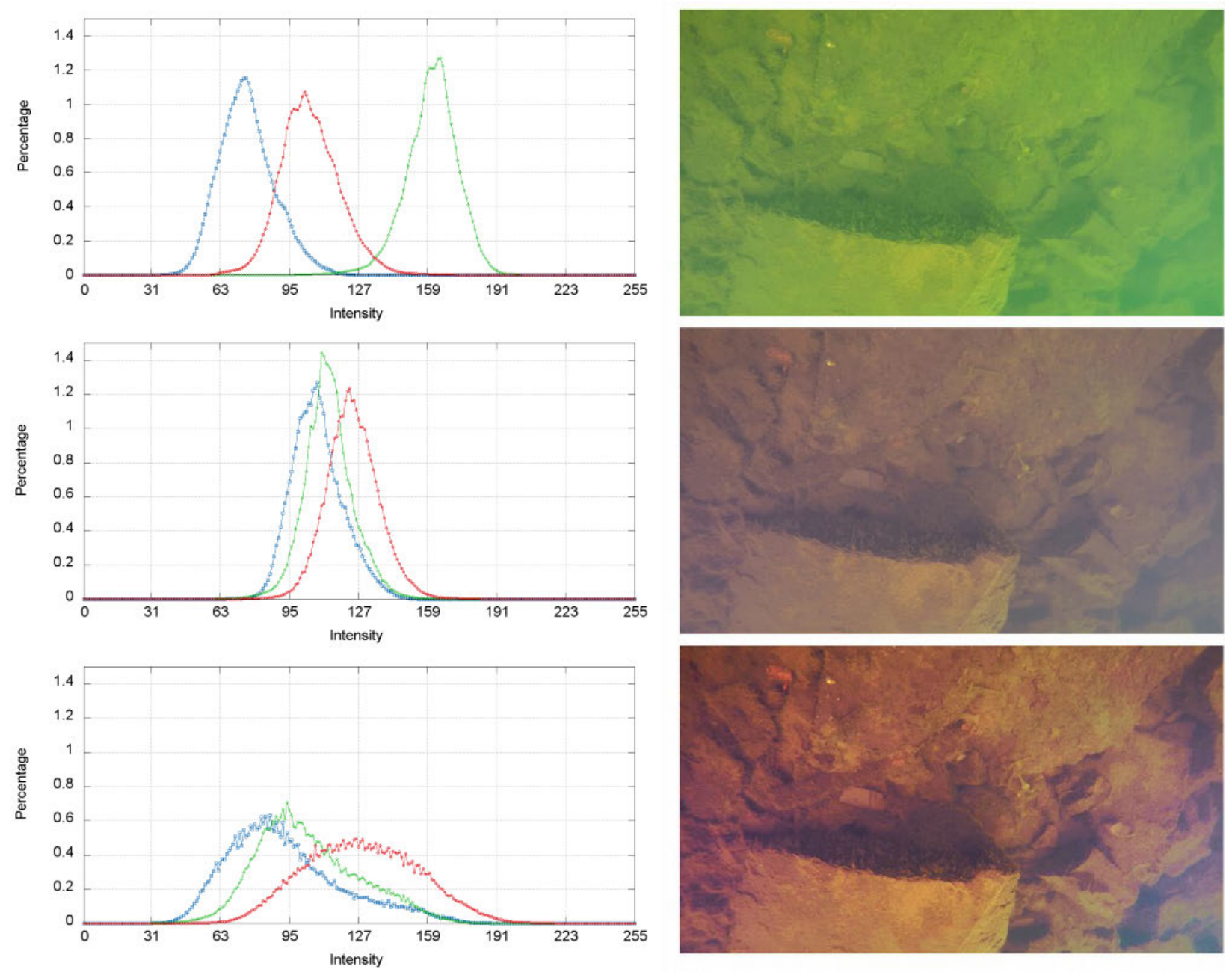

Figure 16. This image was taken in a pool in Kamenz/Germany. The first row shows the original image and the corresponding histogram taken by a GoPro Hero 4 BE with 1080p. The second row shows the result after subtraction of the estimated water color and the third after increasing the contrast. 

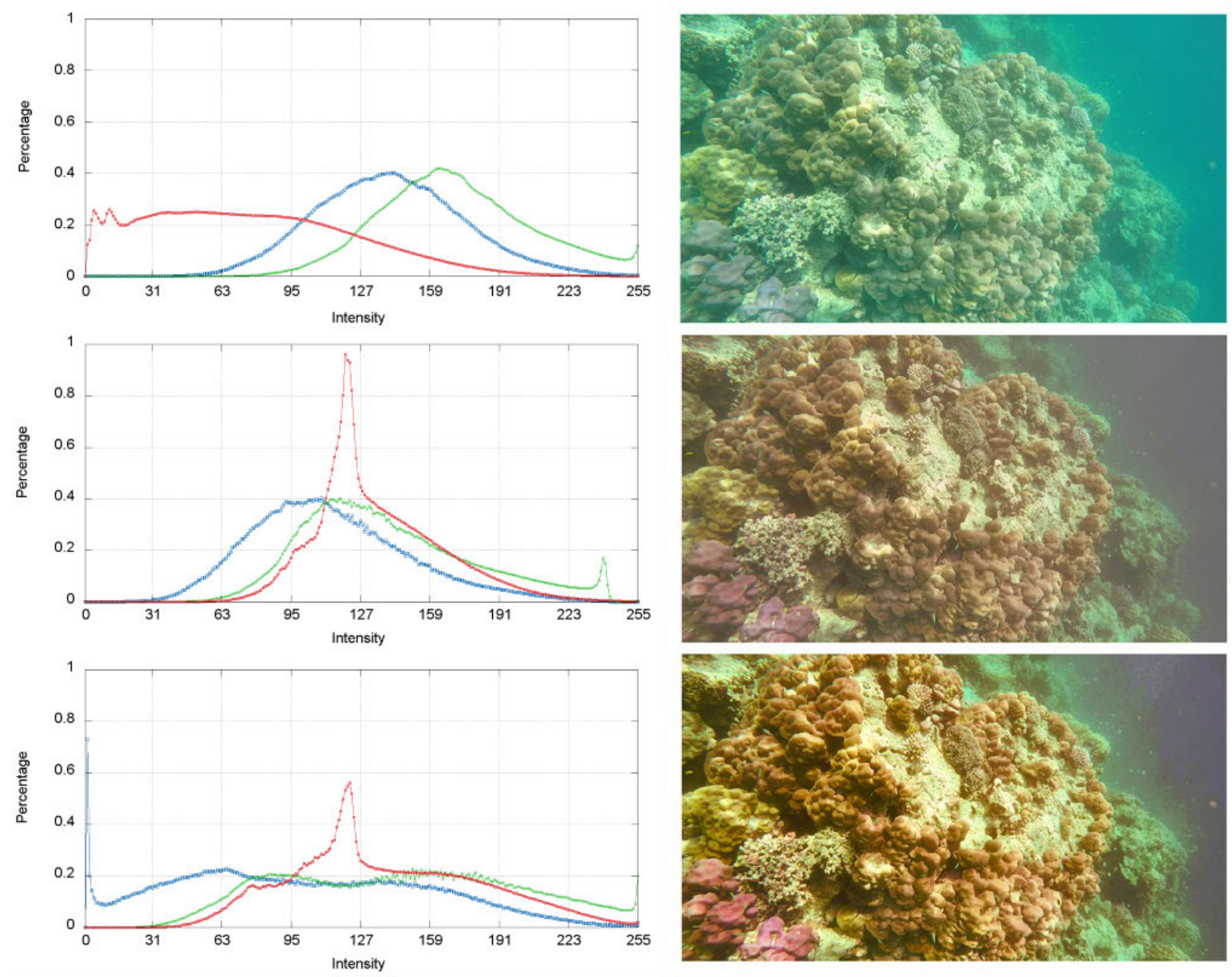

Figure 17. This image was taken in Surin Islands/Thailand by André Knuth. The first row shows the original image and the corresponding histogram taken by a GoPro Hero 4 BE with 1080p. The second row shows the result after subtraction of the estimated water color and the third after increasing the contrast. 

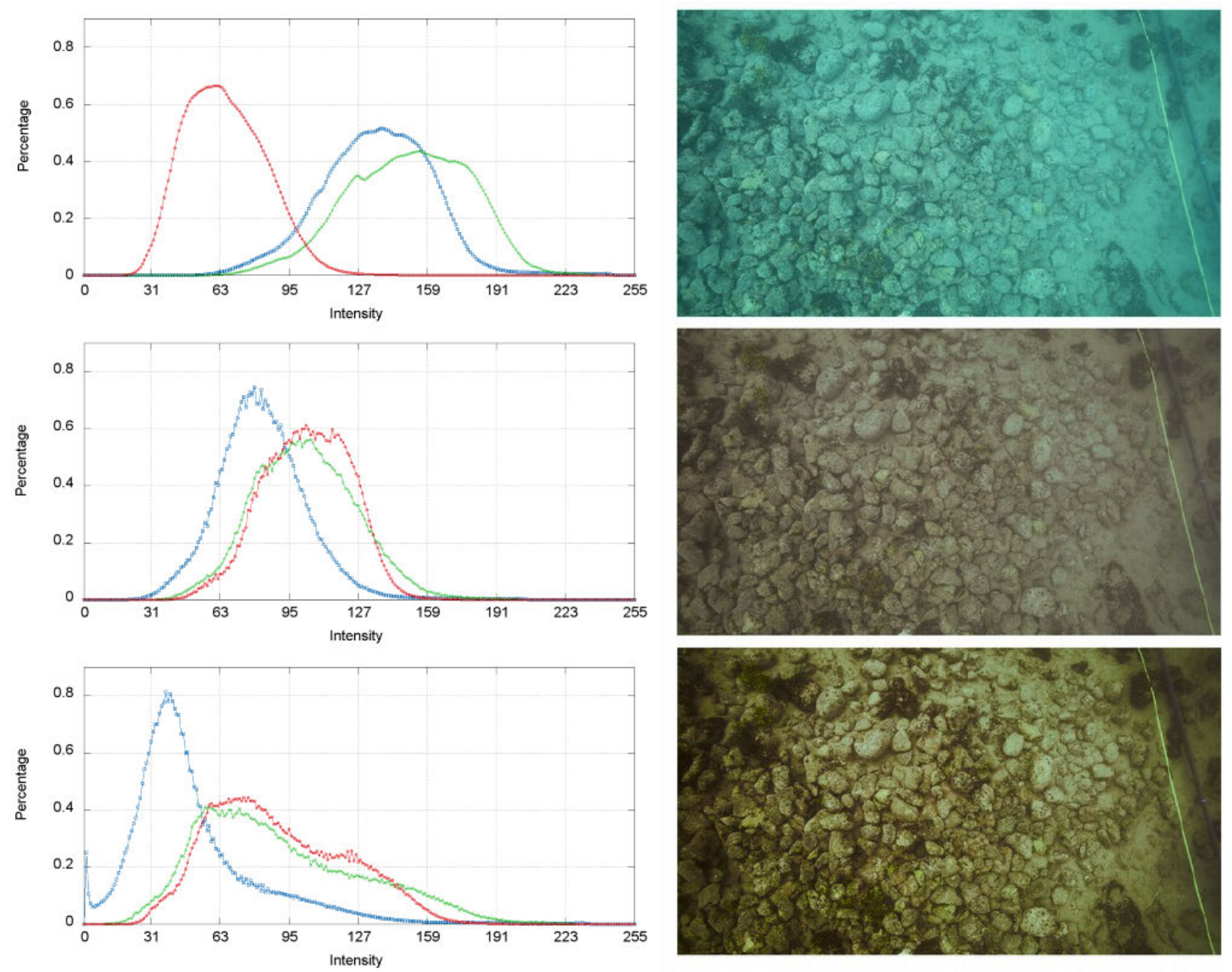

Figure 18. This image was taken in Veruda/Croatia. The first row shows the original image and the corresponding histogram taken by a GoPro Hero 4 BE with 1080p. The second row shows the result after subtraction of the estimated water color and the third after increasing the contrast.

Received March 2017; revised July 2017; accepted August 2017. 